23

\title{
Анализ оптических свойств однородных металлических, окисных наночастиц и двухслойных наночастиц с металлическим ядром и окисной оболочкой с целью эффективного поглощения солнечной радиации
}

\author{
(C) Л.Г. Астафььева ${ }^{1}$, В.К. Пустовалов ${ }^{2}$, В. Фритче ${ }^{3}$ \\ ${ }^{1}$ Институт ффизики им. Б.И. Степанова НАН Беларуси, \\ 220072 Минск, Беларусь \\ ${ }^{2}$ Белорусский национальный технический университет, \\ 220013 Минск, Беларусь \\ ${ }^{3}$ Институт фотонных технологий (IPHT), \\ 07745 Иена, Германия \\ e-mail: astafev@dragon.bas-net.by
}

Поступила в редакцию 26.03.2018 г.

В окончательной редакции 26.10.2018 г.

Принята к публикации 06.11.2018 г.

\begin{abstract}
В последнее время активно изучаются вопросы использования наночастиц для поглощения солнечной радиации и фототермических нанотехнологий. Эффективность применения наночастиц в качестве фототермических агентов для солнечной энергии определяется спектральными оптическими свойствами наночастиц. Выполнено компьютерное моделирование оптических свойств однородных металлических (никелевых, титановых, молибденовых) наночастиц, их окислов и наночастиц, состоящих из металлического ядра и окисной оболочки, с радиусами в диапазоне $50-100 \mathrm{~nm}$ в спектральном интервале $200-2500 \mathrm{~nm}$. Исследовано влияние радиусов наночастиц, типов металлов и их окислов на спектральные коэффициенты эффективности поглощения $K_{\mathrm{abs}}$ и рассеяния $K_{\text {sca }}$ излучения наночастицами. Выбор подходящего типа наночастиц для поглощения солнечного излучения был проведен на основе сравнительного анализа зависимостей коэффициентов эффективности поглощения $K_{\mathrm{abs}}$, интенсивности солнечного излучения $I_{S}$ и параметра $P_{1}=K_{\mathrm{abs}} / K_{\mathrm{sca}}$ от длины волны. Сферические двухслойные наночастицы, состоящие из никеля, титана в ядре и окисных оболочек, с радиусами около $75,100 \mathrm{~nm}$ могут быть применены в спектральном интервале 200-2500 nm для эффективного поглощения солнечной радиации. Эти результаты вносят существенный вклад в изучение оптических свойств наночастиц, которые могут быть использованы в системах тепловой энергии.
\end{abstract}

DOI: $10.21883 /$ OS.2019.03.47381.88-18

\section{Введение}

В последние годы были исследованы вопросы применения наночастиц для поглощения солнечной радиации, для фототермической нанотехнологии и солнечной тепловой энергетики. Для повышения поглощения при использования солнечного света [1-5], для фотокатализа [6-8], для энергетических применений [9-18] используются различные наночастицы в наножидкостях. Поверхностный плазмонный резонанс определяет эффективность поглощения и рассеяния излучения наночастицами [19-22]. Максимальное улавливание солнечной энергии и последующее преобразование в тепловую энергию будут реализованы в случае высокого поглощения излучения наночастицами. Радиационное рассеяние приводит к уменьшению эффективности процессов поглощения и преобразования света в теплоту.

Металлические наночастицы представляют особый интерес для применения солнечной энергии и нанотехнологий. Оптические свойства металлических наночастиц $\mathrm{Cu}, \mathrm{Au}, \mathrm{Al}, \mathrm{Ag}$ и т. д. были исследованы с целью при- менения в нанотехнологиях в спектральном интервале 300-1000 nm [20,22-24]. К сожалению, металлические наночастицы Ti, Ni, Мо не исследовались в широких интервалах радиусов наночастиц и длин волн излучения 200-2500 nm.

Исследование двухслойных наночастиц, состоящих из металлического ядра и окисной оболочки, также очень интересно для улучшения и манипуляции резонансами плазменного спектра наночастиц в дополнение к чистым металлическим наночастицам. Образование окисной оболочки на металлической наночастице может быть достигнуто различными химическими [25] и физическими $[26,27]$ методами и в результате естественного окисления металлических наночастиц в реактивной среде. Естественное окисление чистых металлических наночастиц в газообразных или жидких средах, содержащих компоненты кислорода (воздух, вода, пар), приводит к образованию тонкой окисной оболочки толщиной около 5-10 nm на металлических наночастицах. Действие интенсивного оптического (солнечного) излучения и нагревания наночастиц может способствовать окисле- 
нию поверхностного слоя металлической наночастицы и образованию двухслойных металлических/окисных наночастиц с ядром-оболочкой.

Оптические свойства наночастиц с металлическим ядром и его окисной оболочкой $\left(\mathrm{Ag}-\mathrm{Ag}_{2} \mathrm{O}, \mathrm{Al}-\mathrm{Al}_{2} \mathrm{O}_{3}, \mathrm{Cu}-\right.$ $\left.\mathrm{Cu}_{2} \mathrm{O}\right)[28,29]$ не использовались для целей поглощения солнечного излучения. В экспериментальных и теоретических исследованиях поглощения солнечной радиации использовались гомогенные окисные наночастицы $\left(\mathrm{Al}_{2} \mathrm{O}_{3}, \mathrm{SiO}_{2}, \mathrm{CuO}, \mathrm{ZnO}\right.$ и др.) [12-14,16]. К сожалению, нет достаточных оснований для использования перечисленных окисных наночастиц для поглощения солнечной энергии.

Существующий разрыв в знаниях между опубликованными результатами и потребностями в наночастицах с соответствующими оптическими свойствами явно требует исследований и выбора оптических свойств потенциально подходящих наночастиц, которые могут быть применены для эффективного поглощения и преобразования солнечной энергии в прикладных системах тепловой энергетики.

Сравнительный анализ оптимальных параметров различных металлических наночастиц, двухслойных металлических/окисных наночастиц и чистых окисных наночастиц для их использования в качестве агентов для поглощения солнечной энергии и в прикладных нанотехнологиях все еще отсутствует. Наши исследования направлены на изучение оптических свойств наночастиц для применения в прикладных энергетических системах для эффективного поглощения энергии солнечного излучения. Здесь мы представляем результаты комплексного исследования оптических свойств сферических металлических, окисных и металлическое ядро-окисная оболочка наночастиц, размещенных в воде, на основе компьютерного моделирования для их применения в поглощении солнечного излучения.

\section{Оптические свойства наночастиц в спектральном интервале 200-2500 nm}

Достижение максимального поглощения и минимального рассеяния излучения наночастицами очень важно для эффективного захвата солнечной энергии. Факторы эффективности поглощения $K_{\mathrm{abs}}$, рассеяния $K_{\mathrm{sca}}$ и экстинкции $K_{\mathrm{ext}}=K_{\mathrm{abs}}+K_{\mathrm{sca}}$ излучения с длиной волны $\lambda$ описывают оптические свойства сферической наночастицы с радиусом $r_{0}$ [19]. Параметр $P_{1}=K_{\text {abs }} / K_{\text {sca }}$ должен быть больше $1\left(P_{1}>1\right.$ или $\left.P_{1} \gg 1\right)$, а фактор поглощения $K_{\mathrm{abs}}-$ больше (или намного больше в благоприятных случаях), чем фактор рассеяния $K_{\text {sca. }}$. Такая ситуация позволяет достичь максимальной эффективности взаимодействия солнечной радиации с наночастицей для ее нагрева [30,31]. Рассеяние солнечной радиации наночастицами будет преобладать над поглощением для $P_{1}<1$, и после многократного рассеяния излучения на наночастицах приведет к значительному уменьшению сбора солнечной энергии.

Компьютерное моделирование факторов эффективности поглощения $K_{\mathrm{abs}}$, рассеяния $K_{\mathrm{sca}}$ и экстинкции $K_{\mathrm{ext}}$ солнечной радиации в зависимости от длины волны $\lambda$ в спектральном интервале 200-2500 nm однородными металлическими (Ti, $\mathrm{Ni}, \mathrm{Mo})$, окисными $\left(\mathrm{TiO}_{2}\right.$, $\left.\mathrm{NiO}, \mathrm{MoO}_{3}\right)$ наночастицами и наночастицами с металлическим ядром и окисной оболочкой $\left(\mathrm{Ti}^{-\mathrm{TiO}}{ }_{2}, \mathrm{Ni}-\right.$ $\mathrm{NiO}, \mathrm{Mo}^{-\mathrm{MoO}_{3}}$ ) было проведено на основе обобщенной теории Ми [19]. Использовались однородные наночастицы с радиусами в диапазоне $r_{0}=50-100 \mathrm{~nm}$ и двухслойные наночастицы с ядрами с радиусами в диапазоне $r_{0}=40-90 \mathrm{~nm}$ и толщиной окисной оболочки $\Delta r_{1}=10 \mathrm{~nm}$. Значения оптических показателей преломления металлов, окислов и окружающей воды были взяты из [32-35].

Положения $\lambda_{\mathrm{abs}}^{\max }, \lambda_{\mathrm{sca}}^{\max }$, и $\lambda_{\mathrm{ext}}^{\max }$ и максимальные значения факторов эффективности $K_{\mathrm{abs}}^{\max }, K_{\mathrm{sca}}^{\max }, K_{\mathrm{ext}}^{\mathrm{max}}$ на оси $\lambda$ обозначены на рис. 1-3 разными вертикальными линиями - величины $\lambda_{\mathrm{abs}}^{\max }$ максимального значения фактора поглощения $K_{\mathrm{abs}}^{\max }$ обозначаются сплошными линиями, $\lambda_{\mathrm{sca}}^{\max }-$ штриховыми линиями и $\lambda_{\mathrm{ext}}^{\max }-$ штрихпунктирными линиями в случае разных значений $\lambda_{\mathrm{abs}}^{\max }, \lambda_{\mathrm{sca}}^{\max }$, и $\lambda_{\mathrm{ext}}^{\max }$. В некоторых случаях сплошные линии обозначают одновременное расположение всех максимумов факторов эффективности. Горизонтальные штриховые линии обозначают значение $P_{1}=1$ на рис. $1-3(d, h, l)$.

На рис. 1 представлены зависимости факторов эффективности $K_{\text {abs }}, K_{\text {sca }}$ и $K_{\text {ext }}$ и параметра $P_{1}$ от длины волны $\lambda$ для сферических однородных наночастиц Ті и $\mathrm{TiO}_{2}$ с радиусами $r_{0}=50,75,100 \mathrm{~nm}$ и для двухслойных наночастиц $\mathrm{Ti}^{-} \mathrm{TiO}_{2}$ с толщиной оболочки $\Delta r_{1}=10 \mathrm{~nm}$, радиусами ядра $r_{0}=40,65,90 \mathrm{~nm}$. Радиусы однородных наночастиц $r_{0}$ равны соответственно внешним радиусам $r_{1}=r_{0}+\Delta r_{1}$ двухслойных наночастиц с ядром и оболочкой для сравнения результатов в случае эквивалентных наночастиц.

Плазмонный резонанс электромагнитного (солнечного) излучения на наночастицах $\mathrm{Ti}$ определяет его поглощение. Величины $K_{\mathrm{abs}}^{\max }$ расположены при $\lambda_{\text {abs }}^{\max } \sim 500-1000 \mathrm{~nm}$ для наночастиц Ті с $r_{0}=50,75$, $100 \mathrm{~nm}$. При увеличении $r_{0}$ области размещения максимальных значений факторов $K_{\mathrm{abs}}^{\max }, K_{\mathrm{sca}}^{\mathrm{max}}, K_{\mathrm{ext}}^{\mathrm{max}}$ на оси $\lambda$ для наночастиц Ті сдвинуты в сторону больших значений длин волн. Увеличение $r_{0}$ приводит к уменьшению $K_{\mathrm{abs}}^{\max }$ и образованию двух максимальных значений $K_{\mathrm{abs}}^{\max }$ при $r_{0}=100 \mathrm{~nm}$. Значения $K_{\mathrm{abs}}^{\mathrm{max}}$ для наночастиц Тi с $r_{0}=50$, $75 \mathrm{~nm}$ приблизительно больше или равны значениям $K_{\text {sca }}^{\max }$ в спектральном интервале $200-750 \mathrm{~nm}$, а значения $K_{\mathrm{abs}}^{\mathrm{max}}$ больше или намного больше значений $K_{\mathrm{sca}}^{\max }$ для спектрального интервала 750-2500 nm. Значения $K_{\mathrm{abs}}^{\mathrm{max}}$ меньше, чем значения $K_{\mathrm{sca}}^{\max }$ для наночастиц Тi c $r_{0}=100 \mathrm{~nm}$ в спектральном интервале $\sim 200-1000 \mathrm{~nm}$.

Для наночастиц Ті параметр $P_{1}>1$ для $r_{0}=50$, $75 \mathrm{~nm}$ и для всего спектрального интервала $200-2500 \mathrm{~nm} \quad$ и достигает значений $P_{1} \sim 10-50$ в 
$\mathrm{Ti}$
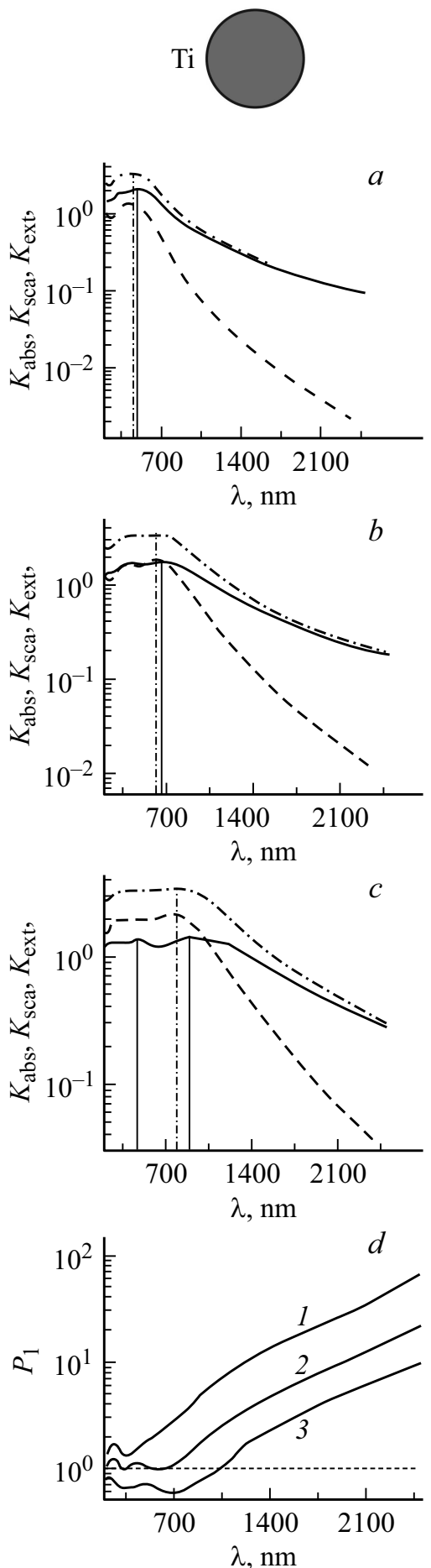
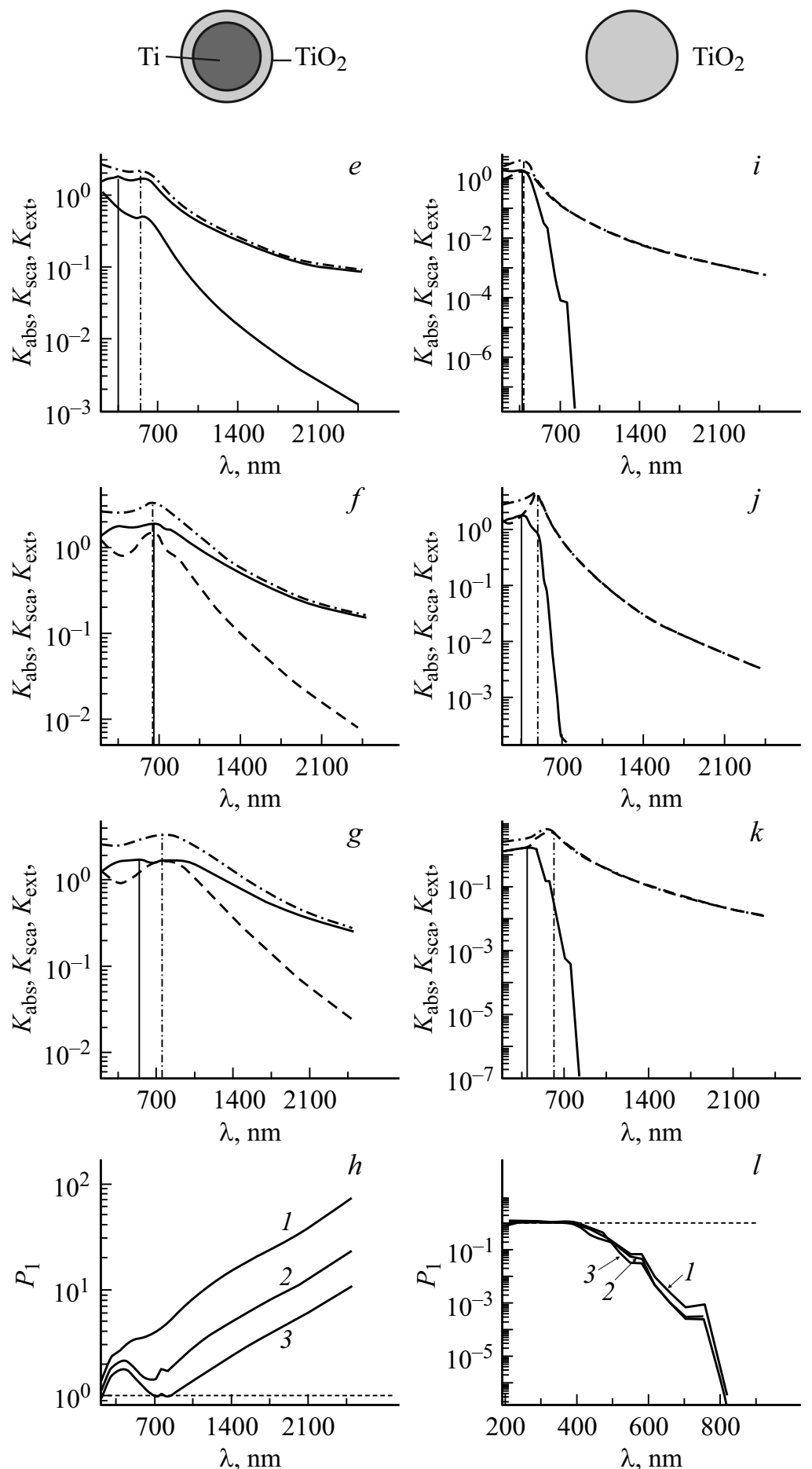

Рис. 1. Зависимости факторов эффективности $K_{\mathrm{abs}}$ (сплошные кривые, $\left.a-c, e-g, i-k\right), K_{\mathrm{sca}}$ (штриховые, $\left.a-c, e-g, i-k\right)$ и $K_{\mathrm{ext}}$ (штрихпунктирные, $a-c, e-g, i-k)$ и зависимости параметра $P_{1}(d, h, l)$ от $\lambda$ для однородных наночастиц Тi $(a-d)$ и ТiO $2(i-l)$ с $r_{0}=50(a, i, d 1, l 1), 75(b, j, d 2, l 2), 100 \mathrm{~nm}(c, k, d 3, l 3)$ и для наночастиц $\mathrm{Ti}^{-\mathrm{TiO}_{2}}$ с $r_{0}=40(e, h 1), 65(f, h 2), 90 \mathrm{~nm}(g, h 3)$ и с $\Delta r_{1}=10 \mathrm{~nm}$.

спектральном интервале $1500-2500 \mathrm{~nm}$ из-за резкого уменьшения $K_{\text {sca }}$ при увеличении $\lambda$. Это означает возможность эффективного применения наночастиц Ті с $r_{0}=50,75 \mathrm{~nm}$ для поглощения солнечного излучения в качестве идеальных поглотителей. Параметр $P_{1}$ меньше 1 при $r_{0}=100 \mathrm{~nm}$ в важном спектральном интервале 200-1000 nm.

Образование окисной оболочки на наночастицах Ті $\left(\mathrm{Ti}+\mathrm{TiO}_{2}\right)$ сдвинуло размещение $\lambda_{\mathrm{abs}}^{\max }$ от $350 \mathrm{~nm}$ при $r_{0}=50 \mathrm{~nm}$ до $670 \mathrm{~nm}$ при $r_{0}=75 \mathrm{~nm}$. Интересно, 

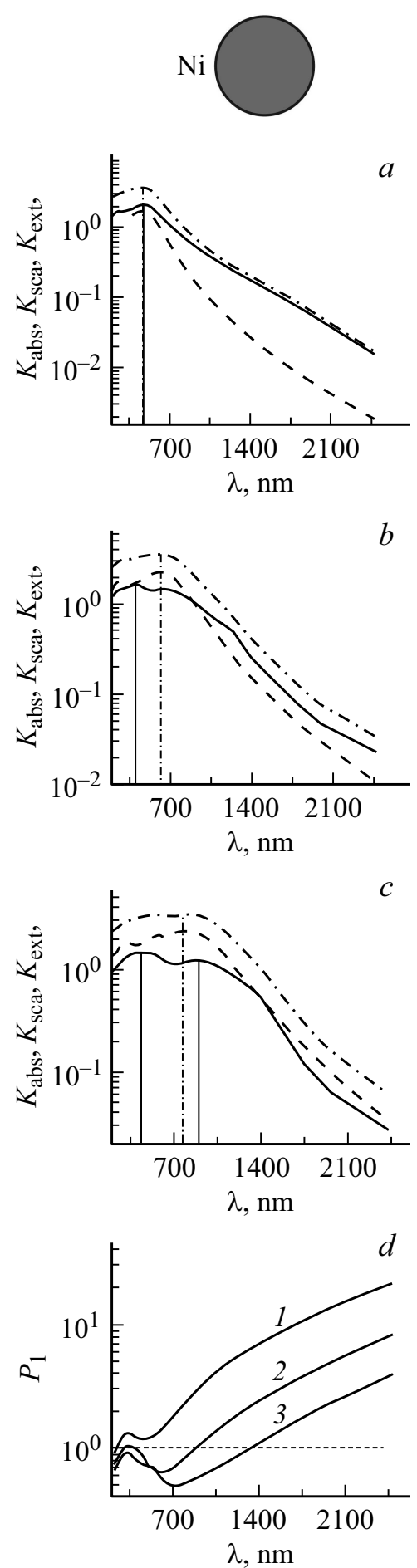
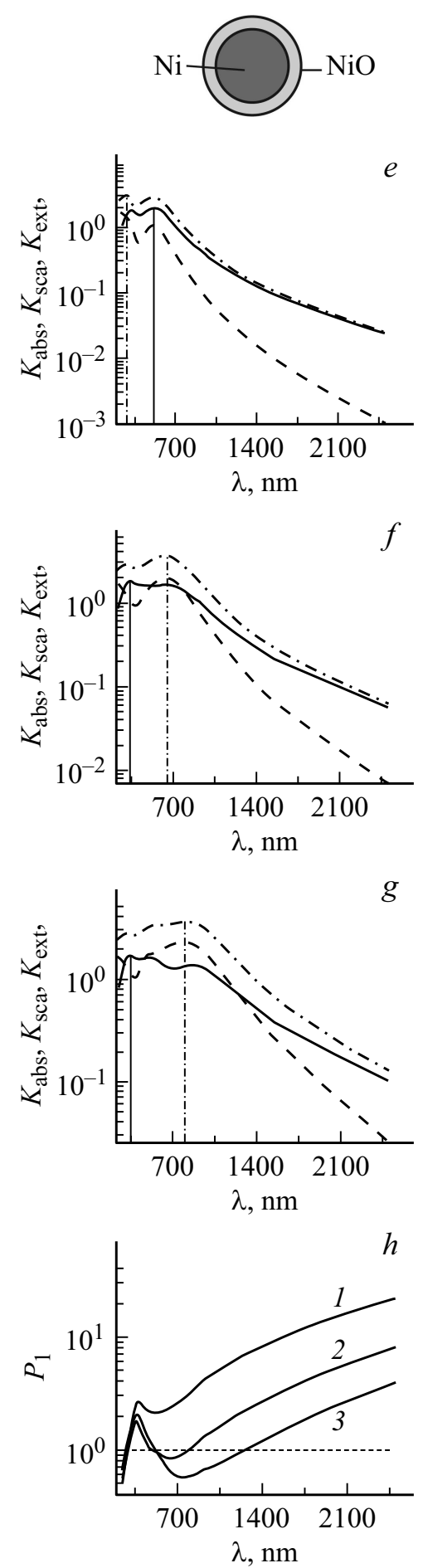
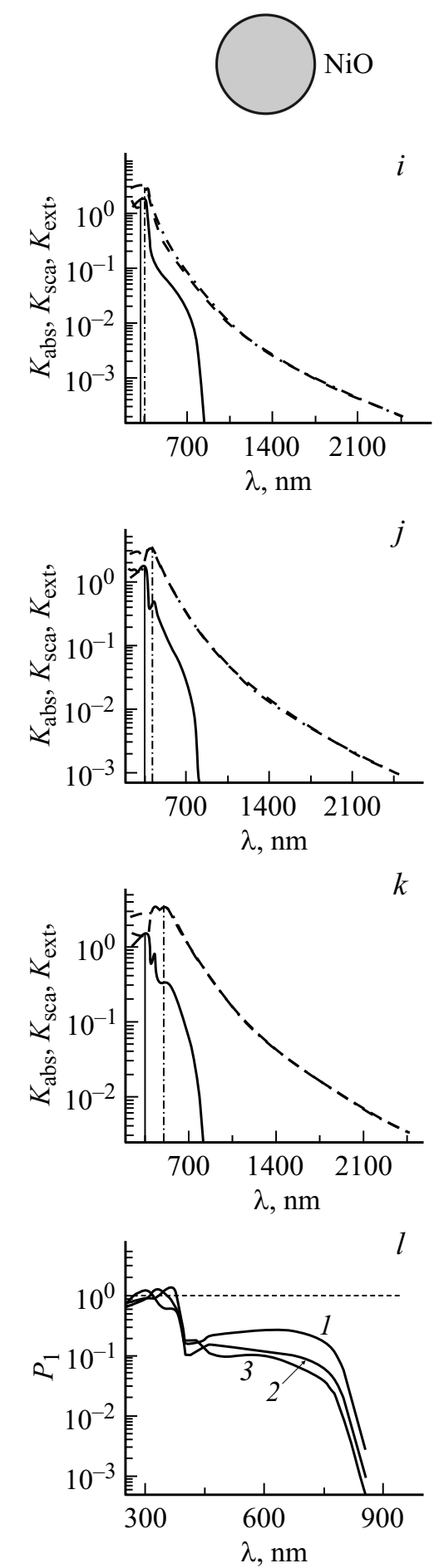

Рис. 2. Зависимости факторов эффективности $K_{\mathrm{abs}}$ (сплошные кривые, $\left.a-c, e-g, i-k\right)$, $K_{\text {sса }}$ (штриховые, $a-c$, $e-g$, $i-k$ ) и $K_{\mathrm{ext}}$ (штрихпунктирные, $a-c, e-g, i-k)$ и зависимости параметра $P_{1}(d, h, l)$ от $\lambda$ для однородных наночастиц $\mathrm{Ni}(a-d)$ и $\mathrm{NiO}(i-l)$ с $r_{0}=50(a, i, d 1, l 1), 75(b, j, d 2, l 2), 100 \mathrm{~nm}(c, k, d 3, l 3)$ и наночастиц Ni-NiO с $r_{0}=40(e, h 1), 65(f, h 2), r_{0}=90 \mathrm{~nm}(c, h 3)$ и с $\Delta r_{1}=10 \mathrm{~nm}$.

что образуются довольно резкий минимум $K_{\mathrm{sca}}^{\min }$ при $\lambda \sim 400 \mathrm{~nm}$ и максимумы $K_{\text {sca }}^{\max }$ при $\lambda \sim 700-800 \mathrm{~nm}$ для $r_{0}=65,90 \mathrm{~nm}$. Значения $K_{\mathrm{abs}}$ больше, чем значения

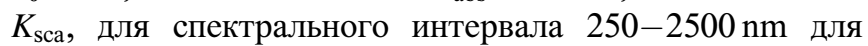
всех представленных значений $r_{0}$ и $\Delta r_{1}$. Следует также отметить резкое уменьшение $K_{\text {sca }}$ с увеличением $\lambda$ в интервале 700-2500 nm.

Мы видим появление максимумов $P_{1}$ для $\lambda \sim 350 \mathrm{~nm}$ для наночастиц $\mathrm{Ti}^{-\mathrm{TiO}_{2}}$ с $r_{0}=40,65,90 \mathrm{~nm}$. Величина $P_{1}$ равна $\sim 1-2$ в спектральном интервале $250-700 \mathrm{~nm}$ 

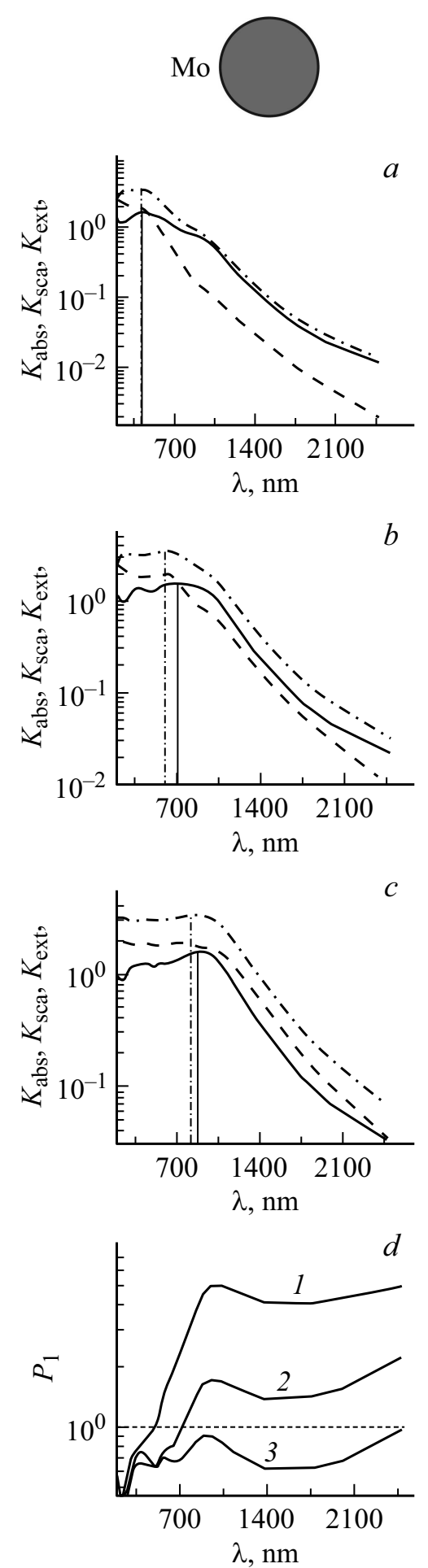
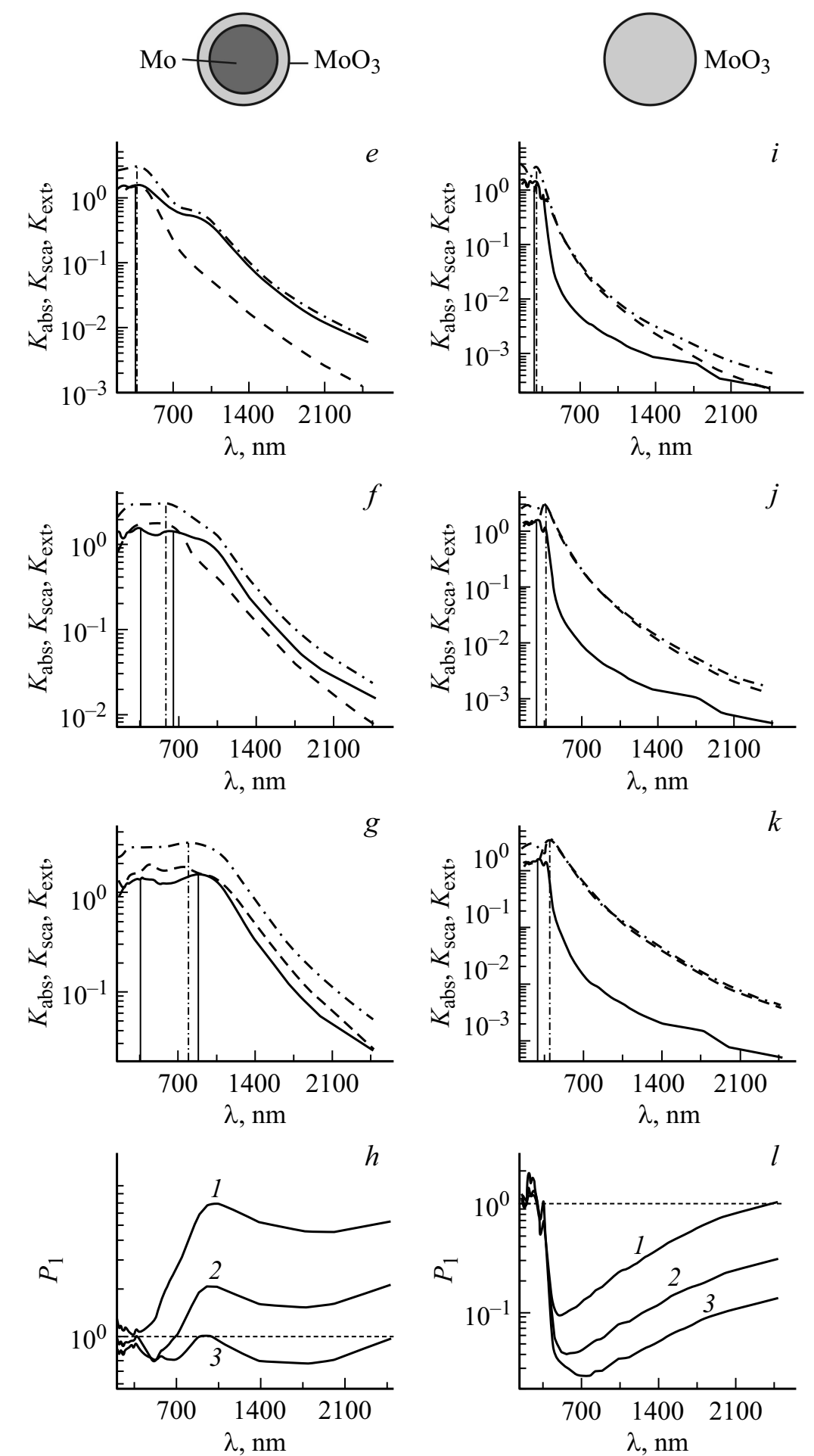

Рис. 3. Зависимости факторов эффективности $K_{\mathrm{abs}}$ (сплошные кривые, $\left.a-c, e-g, i-k\right), K_{\mathrm{sca}}$ (штриховые, $\left.a-c, e-g, i-k\right)$ и $K_{\mathrm{ext}}$ (штрихпунктирные, $a-c, e-g, i-k)$ и зависимости параметра $P_{1}(d, h, l)$ от $\lambda$ для однородных наночастиц Мо $(a-d)$ и МоО $(i-l)$ с $r_{0}=50(a, i, d 1, l 1), 75(b, j, d 2, l 2), 100 \mathrm{~nm}(c, k, d 3, l 3)$ и для наночастиц Мо-МоО с $r_{0}=40(e, h 1), 65(f, h 2), 90 \mathrm{~nm}(c, h 3)$ и с $\Delta r_{1}=10 \mathrm{~nm}$.

и резко возрастает до $P_{1} \sim 10-50$ с увеличением $\lambda$ в спектральном интервале $700-2500 \mathrm{~nm}$. Наличие окисной оболочки приводит к увеличению поглощения при воздействии излучения на двухслойные наночастицы и улучшению возможности применения двухслойных наночастиц $\mathrm{Ti}^{-} \mathrm{TiO}_{2}$.

Значение $K_{\text {abs }}$ для наночастиц $\mathrm{TiO}_{2}$ резко падает до 5-6 порядков величины при увеличении длины волны 
до $\sim 700 \mathrm{~nm}$, ее значение практически равно нулю при $\lambda>700 \mathrm{~nm}$. Это означает, что наночастицы $\mathrm{TiO}_{2}$ обладают значительной поглощательной способностью только в спектральном интервале 200-450 nm. Окисные наночастицы $\mathrm{TiO}_{2}$ практически не поглощают излучение в спектральном интервале $500-2500 \mathrm{~nm}$, содержащем около $85 \%$ всей солнечной энергии. В этом спектральном интервале 500-2500 nm рассеяние наночастицами преобладает над поглощением излучения, а зависимости $K_{\text {sca }}(\lambda)$ и $K_{\text {ext }}(\lambda)$ практически совпадают друг с другом, $K_{\text {sca }}(\lambda) \approx K_{\text {ext }}(\lambda)$ (рис. 1$)$. Параметр $P_{1}$ резко уменьшается с увеличением $\lambda$ при $\lambda>500 \mathrm{~nm}$. Эти результаты показывают практическую невозможность использования окисных наночастиц $\mathrm{TiO}_{2}$ для эффективного поглощения солнечной радиации в спектральном интервале $500-2500 \mathrm{~nm}$.

На рис. 2, 3 представлены зависимости факторов эффективности $K_{\mathrm{abs}}, K_{\mathrm{sca}}$ и $K_{\text {ext }}$ излучения и параметра $P_{1}$ от длины волны $\lambda$ для сферических однородных наночастиц $\mathrm{Ni}, \mathrm{NiO}$ и $\mathrm{Mo}, \mathrm{MoO}_{3}$ соответственно с радиусами $r_{0}=50,75,100 \mathrm{~nm}$ и двухслойных наночастиц $\mathrm{Ni}-\mathrm{NiO}$ и $\mathrm{Mo}^{-\mathrm{MoO}_{3}}$ с ядром и оболочкой с радиусами ядра $r_{0}=40,65,90 \mathrm{~nm}$ и толщиной оболочки $\Delta r_{1}=10 \mathrm{~nm}$.

Фактор эффективности поглощения $K_{\text {abs }}$ для наночастиц $\mathrm{Ni}$ (рис. 2) имеет два слабо выделенных максимума при $r_{0}=100 \mathrm{~nm}$. Параметр $P_{1}$ больше 1 при $r_{0}=50 \mathrm{~nm}$ для спектрального интервала излучения $\lambda=200-2500 \mathrm{~nm}$, он достигает значений $P_{1} \sim 10$ с увеличением $\lambda$ в инфракрасной (ИК) области. Параметр $P_{1}$ меньше 1 в интервале $200 \mathrm{~nm}<\lambda<850 \mathrm{~nm}$ для наночастиц $\mathrm{Ni}$ с $r_{0}=75 \mathrm{~nm}$ и в интервале $300 \mathrm{~nm}<\lambda<1400 \mathrm{~nm}$ при $r_{0}=100 \mathrm{~nm}$. Наночастицы Ni являются хорошими поглотителями излучения во всем оптическом спектре при $r_{0}=50 \mathrm{~nm}$ и в ИК оптическом интервале при $r_{0}=75 \mathrm{~nm}$.

Значения $K_{\text {abs }}$ больше, чем $K_{\text {sca }}$, для наночастиц $\mathrm{Ni-}$ $\mathrm{NiO}$ с $r_{0}=40 \mathrm{~nm}$ в полном спектральном интервале 250-2500 nm. С другой стороны, для наночастиц $\mathrm{Ni}$ $\mathrm{NiO}$ с $r_{0}=65 \mathrm{~nm} K_{\mathrm{abs}}<K_{\mathrm{sca}}$ в спектральном интервале $550-750 \mathrm{~nm}$ и для $r_{0}=90 \mathrm{~nm} K_{\mathrm{abs}}<K_{\text {sca }}$ для спектрального интервала $550-1400 \mathrm{~nm}$. Зависимость параметра $P_{1}$ от $\lambda$ имеет сложный характер для всех представленных значений $r_{0}$ со сформированными максимумами $P_{1} \sim 2-3$ при $\lambda \sim 400 \mathrm{~nm}$.

Зависимость $K_{\mathrm{abs}}$ от $\lambda$ для однородных окисных наночастиц $\mathrm{NiO}$ примерно одинакова в ультрафиолетовом интервале 200-400 nm, и она резко уменьшается на $\sim 3-4$ порядка величины при увеличении $\lambda$, и при $\lambda>700 \mathrm{~nm}$ значение $K_{\mathrm{abs}}$ практически равно 0 . Это поведение определяется зависимостью показателя поглощения $\mathrm{NiO}$ от $\lambda$ [34]. Значения $K_{\text {sca }} \gg K_{\text {abs }}$ и $P_{1} \ll 1$ для спектрального интервала $400-2500 \mathrm{~nm}$, и наночастицы $\mathrm{NiO}$ не подходят для применения в поглощении солнечной энергии.

Спектральная зависимость $K_{\text {abs }}$ для наночастиц Мо (рис. 3) имеет один максимум $K_{\mathrm{abs}}^{\mathrm{max}}$, который смещается от $\lambda_{\mathrm{abs}}^{\mathrm{max}}=380 \mathrm{~nm}$ для $r_{0}=50 \mathrm{~nm}$ до $r_{0}=860 \mathrm{~nm}$ при $r_{0}=100 \mathrm{~nm}$. Фактор поглощения $K_{\mathrm{abs}}$ больше, чем фактор рассеяния $K_{\text {sca }}$, в спектральном интервале $400-2500 \mathrm{~nm}$ при $r_{0}=50 \mathrm{~nm}$ и в интервале $700-2500 \mathrm{~nm}$ для наночастиц Мо с $r_{0}=75 \mathrm{~nm}$. Параметр $P_{1}$ возрастает до значений $P_{1} \sim 5$ при $r_{0}=50 \mathrm{~nm}$ и $P_{1} \sim 2$ при $r_{0}=75 \mathrm{~nm}$ с увеличением $\lambda$. Параметр $P_{1}$ меньше 1 для $r_{0}=50,75 \mathrm{~nm}$ в спектральном интервале $\lambda \sim 200-500 \mathrm{~nm}$ и $\lambda \sim 200-700 \mathrm{~nm}$ соответственно. Это означает, что возможность применения наночастиц Мо для поглощения солнечного излучения мала в упомянутых спектральных интервалах. Для наночастиц Мо $K_{\text {abs }}$ с $r_{0}=100 \mathrm{~nm}$ меньше фактора рассеяния $K_{\text {sca }}$, и $P_{1}<1$ для всего интервала 200-2500 nm.

Влияние образования окисной оболочки на металлические наночастицы с равными радиусами $r_{1}=r_{0}+\Delta r_{1}$ приводит к следующим последствиям. Максимумы плазмонного резонанса создаются и сдвигаются в сторону больших значений длины волны с увеличением $r_{0}$. Это приводит к уменьшению факторов $K_{\mathrm{abs}}^{\mathrm{max}}$ и небольшому влиянию на все оптические факторы при $r_{0}=40-90 \mathrm{~nm}$. Значения параметра $P_{1}$ уменьшаются с ростом $r_{0}\left(r_{1}\right)$. Образование окисной оболочки на металле наночастицы приводит к небольшому увеличению $P_{1}$ в выбранном спектральном интервале для всех значений $r_{0}$. Увеличение $r_{0}$ для однородных наночастиц и $r_{1}$ для наночастиц ядро/оболочка приводит к увеличению $K_{\text {sca }}, K_{\text {ext }}$ по сравнению с $K_{\text {abs. }}$.

$K_{\text {abs }}$ для однородных окисных наночастиц $\mathrm{MoO}_{3}$ резко уменьшается на $\sim 3-4$ порядка величины при увеличении $\lambda$. Значения $K_{\text {sca }} \gg K_{\text {abs }}$ и $P_{1} \ll 1$ для спектрального интервала 400-2500 nm и наночастицы $\mathrm{MoO}_{3}$ не подходят для применения в поглощении солнечной энергии.

На рис. 4 представлены зависимости интенсивности солнечного облучения $I_{S}[36]$ и фактора эффективности поглощения $K_{\mathrm{abs}}$ для наночастиц $\mathrm{Ti}, \mathrm{Ti}^{-\mathrm{TiO}_{2}}, \mathrm{TiO}_{2}, \mathrm{Ni}, \mathrm{Ni}-$ $\mathrm{NiO}, \mathrm{NiO}, \mathrm{Mo}, \mathrm{Mo}-\mathrm{MoO}_{3}, \mathrm{MoO}_{3}$ от длины волны для $r_{0}$ $r_{1}=50,75,100 \mathrm{~nm}$.

Нагрев наночастиц (повышение температуры $\Delta T_{0}$ наночастиц при сравнении с его исходным значением) [30,31] оптическим (солнечным) излучением в спектральном интервале $\lambda_{1}-\lambda_{2}=200-2500 \mathrm{~nm}$ пропорционален (для двухслойных наночастиц $r_{0}$ заменяется на $r_{1}$ в приведенных ниже формулах)

$$
\Delta T_{0} \sim r_{0} \int_{\lambda_{1}}^{\lambda_{2}} I_{S}(\lambda) K_{\mathrm{abs}}\left(r_{0}, \lambda\right) d \lambda .
$$

Максимальная эффективность нагревания наночастиц достигается, когда правая часть уравнения (1) имеет максимальное значение. Следует отметить, что зависимость $I_{S}(\lambda)$ (рис. 1-3) имеет некоторую фиксированную форму и может быть смоделирована зависимостью излучения черного тела от $\lambda$ [36]. Очевидно, что для достижения максимального значения интеграла в (1) зависимость $K_{\mathrm{abs}}(\lambda)$ должна иметь приблизительно вид, 


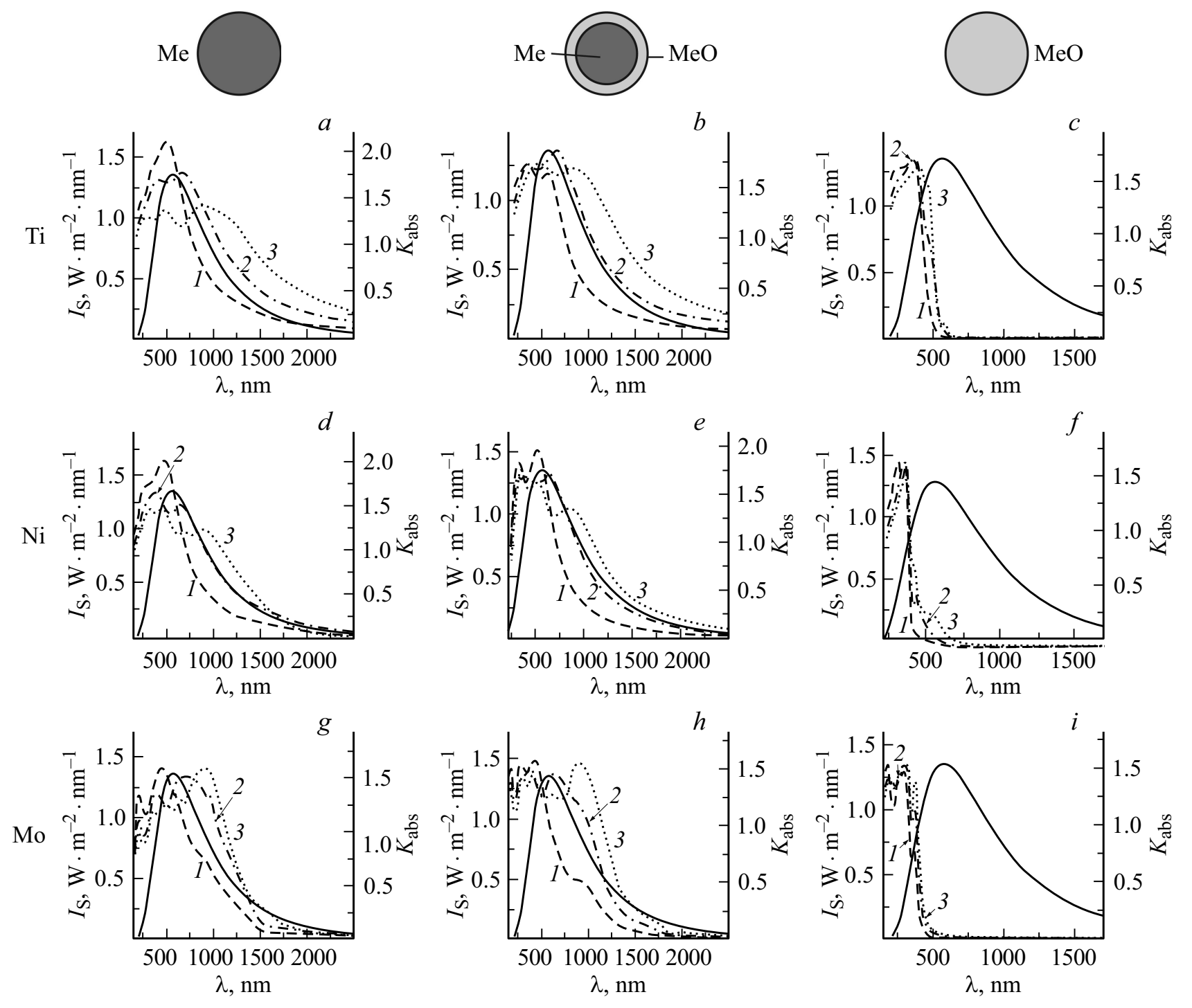

Рис. 4. Зависимости солнечного излучения $I_{S}\left(a-i\right.$, сплошные линии, левая ось) и фактора эффективности поглощения $K_{\text {abs }}(a-i$, правая ось) от $\lambda$ для наночастиц с радиусами $r_{0}, r_{1}=50$ (пунктирные линии), 75 (штриховые), $100 \mathrm{~nm}$ (штрихпунктирные) и наночастиц $\mathrm{Ti}(a), \mathrm{Ti}_{-} \mathrm{TiO}_{2}(b), \mathrm{TiO}_{2}(c), \mathrm{Ni}(d), \mathrm{Ni}-\mathrm{NiO}(e), \mathrm{NiO}(f), \mathrm{Mo}(g), \mathrm{Mo-}-\mathrm{MoO}_{3}(h), \mathrm{MoO}_{3}(i)$. Введены обозначения: Ме металл, $\mathrm{O}$ - окисел.

аналогичный зависимости $I_{S}(\lambda)$, с возможным небольшим сдвигом от положения максимальной интенсивности солнечного излучения. Максимальное значение $\Delta T_{0}^{\max }$ может быть реализовано с использованием максимального соответствующего значения $r_{0}^{\max }$ и максимальных значений $K_{\mathrm{abs}}^{\max }\left(r_{0}^{\max }, \lambda\right)$ для всех $\lambda$ во всем спектральном интервале $\lambda_{2}-\lambda_{1}$, если это возможно:

$$
\Delta T_{0}^{\max } \sim r_{0}^{\max } \int_{\lambda_{1}}^{\lambda_{2}} I_{S}(\lambda) K_{0}^{\max }\left(r_{0}^{\max }, \lambda\right) d \lambda .
$$

Цель реализации $\Delta T_{0}^{\max }$ может быть достигнута путем выбора типа наночастиц и их размера, материалов ядра и оболочки и т.д.

Для наночастиц Ті зависимости $K_{\mathrm{abs}}$ от $\lambda$ при $r_{0}=75$, $100 \mathrm{~nm}$ близки к зависимости $I_{S}(\lambda)$, а значения $K_{\mathrm{abs}}$ больше, чем при $r_{0}=50 \mathrm{~nm}$, в спектральном интервале $\lambda \sim 600-2500 \mathrm{~nm} \mathrm{c} K_{\mathrm{abs}}^{\max } \approx 1.75$ и в спектральном интервале $\lambda \sim 750-2500 \mathrm{~nm}$ с $K_{\mathrm{abs}}^{\max } \approx 1.5$. Для наночастиц $\mathrm{Ti}^{-\mathrm{TiO}_{2}}$ зависимость $K_{\mathrm{abs}}$ от $\lambda$ при $r_{0}=65 \mathrm{~nm}$ близка к зависимости $I_{S}(\lambda)$ в спектральном интервале $\lambda \sim 500-2500 \mathrm{~nm} \mathrm{c} K_{\mathrm{abs}}^{\max } \approx 1.85$. Значения $K_{\mathrm{abs}}$ для $r_{0}=90 \mathrm{~nm}$ больше, чем $K_{\text {abs }}$ для $r_{0}=40,65 \mathrm{~nm}$, в спектральном интервале $\lambda \sim 750-2500 \mathrm{~nm}$ и меньше в спектральном интервале $\lambda \sim 400-750 \mathrm{~nm}$.

Такая же ситуация существует для наночастиц $\mathrm{Ni}$ и Ni-NiO. Зависимости $K_{\text {abs }}$ от $\lambda$ при $r_{0}, r_{1}=75 \mathrm{~nm}$ близки к зависимости $I_{S}(\lambda)$ в спектральном интервале $\lambda \sim 400-2500 \mathrm{~nm} \mathrm{с} K_{\mathrm{abs}}^{\max } \approx 1.6$ и $K_{\mathrm{abs}}^{\max } \approx 1.65$ соответственно. Следует отметить, что зависимости $K_{\mathrm{abs}}(\lambda)$ для $r_{0}, r_{1}=100 \mathrm{~nm}$ также довольно близки к зависимости $I_{S}(\lambda)$, и они имеют более высокие значения $K_{\mathrm{abs}}$, 
чем для $r_{0}, r_{1}=75 \mathrm{~nm}$, в спектральном интервале от $\lambda \sim 800-2500 \mathrm{~nm}$.

Для наночастиц Мо и Мо- $\mathrm{MoO}_{3}$ с $r_{0}, r_{1}=75,100 \mathrm{~nm}$ значение $\lambda_{\mathrm{abs}}^{\max } \sim 700,950 \mathrm{~nm}$ было смещено в сторону больших длин волн по сравнению с положением $\lambda_{\mathrm{abs}}^{\max } \approx 530 \mathrm{~nm}$. Но эти наночастицы имеют более высокие значения $K_{\mathrm{abs}}$, чем для $r_{0}, r_{1}=50 \mathrm{~nm}$, в спектральном интервале $\lambda \sim 500-2500 \mathrm{~nm}$. Факторы поглощения $K_{\text {abs }}$ для наночастиц $\mathrm{TiO}_{2}, \mathrm{NiO}, \mathrm{MoO}_{3}$ резко снижаются при увеличении $\lambda$, и они практически равны нулю при $\lambda>500 \mathrm{~nm}$. Эти наночастицы не могут использоваться для эффективного поглощения солнечной радиации.

Исследованные зависимости оптических факторов $K_{\text {abs }}, K_{\text {sca }}$ и $K_{\text {ext }}$ и параметра $P_{1}$ в диапазонах радиусов наночастиц 50-100 nm и в спектральном интервале 200-2500 nm являются новыми и содержат новые данные в области оптических свойств наночастиц. Анализ оптических свойств представленных наночастиц Тi, Ti$\mathrm{TiO}_{2}, \mathrm{Ni}, \mathrm{Ni}-\mathrm{NiO}$ и, возможно, в меньшей степени Мо, $\mathrm{Mo}-\mathrm{MoO}_{3}$ позволяет сделать вывод о том, что эти наночастицы являются подходящими кандидатами для применений в солнечной тепловой энергетике.

\section{Заключение}

Выбор новых оптических свойств и оптимальных параметров гомогенных металлических, окисных и ядрометалл и оболочка-окисел наночастиц для эффективного поглощения солнечной радиации и для термических применений основан на исследованиях материалов и структуры наночастиц, их размеров (радиусов) ит.д. и сравнительном анализе их оптических свойств. Изучение указанных параметров было проведено в настоящей работе в качестве предпосылки для успешного использования наночастиц для применения в солнечной тепловой энергетике.

Исследованы новые зависимости факторов поглощения $K_{\text {abs, }}$, рассеяния $K_{\text {sca }}$ и экстинкции $K_{\text {ext }}$ и параметра $P_{1}$ однородных наночастиц $\mathrm{Ti}, \mathrm{Ni}, \mathrm{Mo}$ и $\mathrm{TiO}_{2}, \mathrm{NiO}$, $\mathrm{MoO}_{3}$ и двухслойных наночастиц ядро-оболочка Ті$\mathrm{TiO}_{2}, \mathrm{Ni}-\mathrm{NiO}, \mathrm{Mo}-\mathrm{MoO}_{3}$ для длин волн в спектральном интервале 200-2500 nm и в диапазоне радиусов наночастиц $r_{0}, r_{1}=50-100 \mathrm{~nm}$ на основе компьютерного моделирования. Параметр $P_{1}$ описывает доминирование поглощения наночастиц над рассеянием, если $P_{1}>1$.

Анализ результатов показывает, что окисные наночастицы $\mathrm{TiO}_{2}, \mathrm{NiO}, \mathrm{MoO}_{3}$ не могут использоваться для эффективного поглощения солнечной радиации изза чрезвычайно низкого поглощения в видимой и ИК областях солнечного оптического спектра, их параметры $P_{1} \ll 1$.

Металлические и ядро-оболочка наночастицы имеют несколько общих особенностей. Увеличение $r_{0}, r_{1}$ сдвигает положение $\lambda_{\mathrm{abs}}^{\max }, \lambda_{\mathrm{sca}}^{\max }, \lambda_{\mathrm{ext}}^{\max }$ и значений $K_{\mathrm{abs}}^{\max }, K_{\mathrm{sca}}^{\max }$, $K_{\mathrm{ext}}^{\mathrm{max}}$ в сторону больших значений $\lambda$ в видимой и ИК областях с уменьшением значения $K_{\mathrm{abs}}^{\mathrm{max}}$ и формированием второго максимума. Металлические наночастицы из Тi, $\mathrm{Ni}$ и наночастицы металлическое ядро-окисная оболочка из $\mathrm{Ti}_{-} \mathrm{TiO}_{2}$ с $r_{0}, r_{1} \sim 75,100 \mathrm{~nm}$ имеют максимальные значения фактора поглощения $K_{\mathrm{abs}}(\lambda)$, и их зависимости $K_{\text {abs }}(\lambda)$ близки к спектральной зависимости солнечной радиации $I_{S}(\lambda)$.

Параметр $P_{1}$ больше 1 для наночастиц Ті и $\mathrm{Ti}^{-\mathrm{TiO}_{2}}$ и наночастиц $\mathrm{Ni}$ и $\mathrm{Ni}-\mathrm{NiO}$ с $r_{0}=50,75 \mathrm{~nm}$ для всего спектрального интервала 200-2500 nm. Но параметр $P_{1}$ уменьшается с увеличением $r_{0}, r_{1}$ и при $r_{0}=100 \mathrm{~nm}$, а в некоторых случаях и при $r_{0}=75 \mathrm{~nm}, P_{1}$ меньше 1 в узких или широких спектральных интервалах и, по крайнем случае, в полном спектральном интервале 200-2500 nm. Параметр $P_{1}$ увеличивается с увеличением $\lambda$ в ИК области спектра до значений $P_{1} \sim 10-50$ для $r_{0}, r_{1}=50,75 \mathrm{~nm}$. Тонкая окисная оболочка толщиной $10 \mathrm{~nm}$ влияет на оптические свойства двухслойных наночастиц в ультрафиолетовом и вблизи видимого спектрального интервала из-за оптического поглощения в этих спектральных областях окисным слоем.

Окончательный выбор лучших наночастиц может быть достигнут путем комплексного анализа свойств наночастиц, который позволяет реализовать максимальное нагревание наночастиц. Исследованные наночастицы могут применяться в качестве хороших поглотителей для эффективного поглощения солнечной радиации в применяемых энергетических тепловых системах (прямые солнечные поглощающие коллекторы).

\section{Список литературы}

[1] Tang Y., Vlahovic B. // Nanoscale Res Lett. 2013. V. 8. P. 65.

[2] Zhang H., Chen H.-J., Du X., Wen D. // Solar Energy. 2014. V. 100. P. 141.

[3] Mlinar V. // Nanotechnology. 2013. V. 24. P. 042001.

[4] Crisostomo F., Jerrild N., Mesgari S., Li Q., Taylor R. // Appl. Energy. 2017. V. 193. P. 1.

[5] Du M., Tang G. // Solar Energy. 2016. V. 137. P. 393.

[6] Hashemi S., Choi J.-W., Psaltis D. // Phys. Chem. Chem. Phys. 2014. V. 16. P. 5137.

[7] Borgesa M., Sierra M., Cuevas E., García R., Esparzad P. // Solar Energy. 2016. V. 135. P. 527.

[8] Duan H., Xuan Y. // Appl. Energy. 2014. V. 114. P. 22.

[9] Kameya Y., Hanamura K. // Solar Energy. 2011. V. 85. P. 299.

[10] He Q., Wang S., Zeng S., Zheng Z. // Energy Conversion Management. 2013. V. 73. P. 150.

[11] Hossain M., Saidur R., Sabri M., Said Z., Hassani S. // Renew. Sustain. Energy Reviews. 2015. V. 43. P. 750.

[12] Hussein A.K. // Renew. Sustain. Energy Reviews. 2016. V. 2. P. 767.

[13] Kasaeian A., Eshghi A., Sameti M. // Renew. Sustain. Energy Reviews. 2015. V. 43. P. 584.

[14] Leong K., Ong H., Amer N., Norazrina M., Risby M. // Renew. Sustain. Energy Reviews. 2016. V. 53. P. 1092.

[15] Jin H., Lin G., Bai L., Amjad M., Filho E., Wen D. // Solar Energy. 2016. V. 139. P. 278.

[16] Yiamsawas T., Mahian O., Dalkilic A., Kaewnai S., Wongwises S. // Appl. Energy. 2013. V. 111. P. 40. 
[17] Amjad M., Raza G., Xin Y., Pervaiz S., Wen D. // Appl. Energy. 2017. V. 206. P. 393.

[18] Chen M., He Y., Zhu J., Wen D.I. // Appl. Energy. 2016. V. 181. P. 65.

[19] Bohren C., Huffman D. Absorption and Scattering of Light by Small Particles. Wiley Interscience, 1983. 545 p.; Борен К., Хафмен Д. Поглощение и рассеяние света малыми частицами. М.: Мир, 1986. 664 с.

[20] Kreibig U., Vollmer M. Optical Properties of Metal Clusters. Heidelberg: Springer, 1995. $532 \mathrm{p}$.

[21] Maier S. Plasmonics: Fundamentals and Applications. Heidelberg: Springer, 2007. $201 \mathrm{p}$.

[22] Pelton M., Aizpurua J., Bryant G. // Laser Photonics. 2008. V. 2. P. 136

[23] Amendola V., Bakr O., Stellacci F. // Plasmonics. 2010. V. 5. P. 85.

[24] Astafyeva L.G., Pustovalov V.K., Fritzsche W. // NanoStructures \& Nano-Objects. 2017. V. 12. P. 57.

[25] Jones M., Osberg K., MacFarlane L., Langille R., Mirkin C. // Chem. Rev. 2011. V. 111. P. 3736.

[26] Pustovalov V., Bobuchenko D. // Int. J. Heat Mass Transfer. 1989. V. 32. P. 3.

[27] Das S., Datta S., Mukhopadhyay A., Pal K, Basu D. // Materials Chem. Phys. 2010. V. 122. P. 574.

[28] Laaksonen K., Suomela S., Puisto S., Rostedt N., AlaNissila T., Nieminen R. // J. Opt. Soc. Am. B: Opt. Phys. 2013. V. 30. P. 338.

[29] Pustovalov V., Astafyeva L. // J. Nanomaterials. 2015. P. ID 812617. doi 10.1155/2015/812617

[30] Pustovalov V. // Laser Phys. 2011. V. 21. P. 906.

[31] Pustovalov V. // RSC Advances. 2016. V. 6. P. 81266.

[32] Refractive index database. 2015. [Электронный ресурс] Режим доступа: $\mathrm{http}: / /$ refractiveindex.info/

[33] Devore J. // J. Opt. Soc. Am. 1951. V. 41. P. 416.

[34] Mahmoud S., Alshomer S., Tarawnh M. // J. Mod. Phys. 2011. V. 2. P. 1178.

[35] Lajaunie L., Bousher F., Dessapt R., Moreau P. // Phys. Rev. B. 2013. V. 88. P. 115141.

[36] ASTM G-173-03-International standard ISO 9845-1, 1992.

[37] Pustovalov V., Astafyeva L., Fritzsche W. // Solar Energy. 2015. V. 122. P. 1334. 